\title{
Bapineuzumab for mild to moderate Alzheimer's disease in two global, randomized, phase 3 trials
}

Rik Vandenberghe ${ }^{1 *}$, Juha O. Rinne ${ }^{2}$, Mercè Boada ${ }^{3}$, Sadao Katayama ${ }^{4}$, Philip Scheltens ${ }^{5}$, Bruno Vellas ${ }^{6}$, Michael Tuchman ${ }^{7}$, Achim Gass ${ }^{8}$, Jochen B. Fiebach ${ }^{9}$, Derek Hill ${ }^{10}$, Kasia Lobello ${ }^{11}$, David Li ${ }^{11}$, Tom McRae ${ }^{12}$, Prisca Lucas ${ }^{13}$, Iona Evans ${ }^{14}$, Kevin Booth ${ }^{11}$, Gerald Luscan ${ }^{13}$, Bradley T. Wyman ${ }^{15}$, Lisa Hua ${ }^{11}$, Lingfeng Yang ${ }^{11}$, H. Robert Brashear ${ }^{16}$, Ronald S. Black ${ }^{11}$ and for the Bapineuzumab 3000 and 3001 Clinical Study Investigators

\begin{abstract}
Background: Our objective was to evaluate the efficacy (clinical and biomarker) and safety of intravenous bapineuzumab in patients with mild to moderate Alzheimer's disease (AD).

Methods: Two of four phase 3, multicenter, randomized, double-blind, placebo-controlled, 18-month trials were conducted globally: one in apolipoprotein E $\varepsilon 4$ carriers and another in noncarriers. Patients received bapineuzumab $0.5 \mathrm{mg} / \mathrm{kg}$ (both trials) or $1.0 \mathrm{mg} / \mathrm{kg}$ (noncarrier trial) or placebo every 13 weeks. Coprimary endpoints were change from baseline to week 78 on the 11-item Alzheimer's Disease Assessment Scale-Cognitive subscale and the Disability Assessment for Dementia.
\end{abstract}

Results: A total of 683 and 329 patients completed the current carrier and noncarrier trials, respectively, which were terminated prematurely owing to lack of efficacy in the two other phase 3 trials of bapineuzumab in AD. The current trials showed no significant difference between bapineuzumab and placebo for the coprimary endpoints and no effect of bapineuzumab on amyloid load or cerebrospinal fluid phosphorylated tau. (Both measures were stable over time in the placebo group.) Amyloid-related imaging abnormalities with edema or effusion were confirmed as the most notable adverse event.

Conclusions: These phase 3 global trials confirmed lack of efficacy of bapineuzumab at tested doses on clinical endpoints in patients with mild to moderate AD. Some differences in the biomarker results were seen compared with the other phase 3 bapineuzumab trials. No unexpected adverse events were observed.

Trial registration: Noncarriers (3000) ClinicalTrials.gov identifier NCT00667810; registered 24 Apr 2008. Carriers (3001) ClinicalTrials.gov identifier NCT00676143; registered 2 May 2008.

Keywords: Alzheimer's disease, Bapineuzumab, Immunotherapy, Amyloid $\beta$, Clinical trial, ARIA-E, Vasogenic edema

\section{Background}

Immunotherapy with monoclonal antibodies has been under investigation as a therapeutic approach to Alzheimer's disease (AD) [1, 2]. Bapineuzumab is a monoclonal antibody specific to the $\mathrm{N}$-terminus of the amyloid $\beta(\mathrm{A} \beta)$ protein designed to decrease plaque

\footnotetext{
* Correspondence: rik.vandenberghe@uzleuven.be

'University Hospitals Leuven, Department of Neurosciences, Alzheimer

Research Centre KU Leuven, Herestraat 49, 3000, Leuven, Belgium

Full list of author information is available at the end of the article
}

formation and promote clearance of $A \beta$ [2-4]. In phase 2 studies in patients with mild to moderate AD, bapineuzumab reduced phosphorylated tau ( $\mathrm{p}$-tau) protein in cerebrospinal fluid (CSF) and ${ }^{11} \mathrm{C}$-Pittsburgh compound $\mathrm{B}(\mathrm{PiB})$ average uptake visualized by positron emission tomography (PET) [5, 6]. The findings provided a rationale for conducting separate trials in apolipoprotein $\mathrm{E}$ (ApoE) $\varepsilon 4$ allele carriers and noncarriers and for limiting the bapineuzumab dose in carriers to minimize risk of amyloid-related imaging abnormalities 
with edema or effusion (ARIA-E; previously termed vasogenic edema) [7].

The bapineuzumab phase 3 development program consisted of four nearly identical phase 3 trials conducted in parallel to evaluate the efficacy and safety of intravenous (IV) bapineuzumab over 18 months in patients with mild to moderate AD. The other two trials reported elsewhere were conducted primarily in the United States: Study 302 in ApoE $\varepsilon 4$ allele carriers (ClinicalTrials.gov identifier NCT00575055) and Study 301 in noncarriers (ClinicalTrials.gov identifier NCT00 574132) [3]. These studies showed no benefit of bapineuzumab on the cognitive or functional endpoints assessed.

Here we report results from the two global phase 3 trials of IV bapineuzumab in ApoE $\varepsilon 4$ carriers (Study 3001, ClinicalTrials.gov identifier NCT00676143) and noncarriers (Study 3000, ClinicalTrials.gov identifier NCT00 667810 ). Both trials were terminated prematurely because of lack of clinical efficacy observed in the 301 and 302 studies [3].

\section{Methods}

\section{Study design}

Studies 3000 and 3001 were multicenter, randomized, double-blind, placebo-controlled, 18-month clinical trials in which investigators evaluated the efficacy and safety of bapineuzumab $0.5 \mathrm{mg} / \mathrm{kg}$ versus placebo (ratio 3:2) in ApoE \&4 carriers (Study 3001) and bapineuzumab $0.5 \mathrm{mg} / \mathrm{kg}, 1.0 \mathrm{mg} / \mathrm{kg}$, or placebo (ratio 3:3:4) in ApoE $\varepsilon 4$ noncarriers (Study 3000). Originally, Study 3000 included a bapineuzumab $2.0 \mathrm{mg} / \mathrm{kg}$ dose, which was discontinued because of a high rate of clinically symptomatic ARIA-E. Patients randomized to the $2.0 \mathrm{mg} / \mathrm{kg}$ group were reassigned to receive $1.0 \mathrm{mg} / \mathrm{kg}$ for the remainder of the study, and the randomization ratio was adjusted accordingly. Allocation of patients to treatment groups using stratified block randomization proceeded through the entering of subject information by the study coordinator or delegate and the dispenser (unblinded pharmacist) into an interactive voice/web response system. The dispenser was then provided with a subject randomization number and treatment assignment, and a confirmatory facsimile was sent to the dispenser. Randomization was stratified by Mini Mental State Examination (MMSE) scores (16-21; 22-26); concomitant cholinesterase inhibitor and/or memantine use; substudy participation; and, in the carrier study, number of copies of ApoE $\varepsilon 4$ allele (one allele; two alleles). Patients received a total of six IV infusions of bapineuzumab or placebo every 13 weeks, with brain magnetic resonance imaging (MRI) monitoring for ARIA-E conducted at 6 weeks after each infusion. There were three biomarker substudies: a brain amyloid PET substudy, a CSF substudy, and a volumetric magnetic resonance imaging (vMRI) substudy. No interim analyses were planned or performed.

\section{Inclusion criteria}

Patients were eligible for enrollment if they were aged $50-88$ years with a diagnosis of probable AD and had an MMSE score of 16-26, inclusive, and a screening MRI scan consistent with AD. Inclusion and exclusion criteria were similar to those used in the 301/302 studies [3] and are listed in Additional file 2.

\section{Outcome measures}

Coprimary efficacy endpoints were change from baseline to week 78 in 11-item Alzheimer's Disease Assessment Scale-Cognitive subscale (ADAS-Cog/11) score and Disability Assessment for Dementia (DAD) total score. Change from baseline to week 78 in Dependence Scale, Clinical Dementia Rating-Sum of Boxes (CDR-SOB), and Neuropsychological Test Battery (NTB) total Zscores were additional endpoints. Prespecified secondary biomarker endpoints included change from baseline to week 71 in PiB-PET global cortical average (GCA) standardized uptake value ratio (SUVr) of five cortical regions of interest (frontal, lateral temporal, parietal, anterior cingulate, posterior cingulate/precuneus), CSF p-tau, and brain volume assessed by brain boundary shift integral (BBSI) on vMRI. PiB-PET and vMRI were read centrally. Human plasma $A \beta_{x-40}$ peptide levels were measured using a validated electrochemiluminescence immunoassay method on a Meso Scale Discovery (Rockville, MD, USA) platform, which had a range of quantification from 50 to $3200 \mathrm{pg} / \mathrm{ml}$ in $100 \%$ matrix with a minimum required sample dilution of $1: 2$ and maximum sample dilution of 1:64. This platform had an interassay precision of $9 \%$ and an intraassay precision of $5 \%$. CSF ${ }^{181}$ phosphotau concentrations were measured using the INNOTEST enzyme-linked immunosorbent assay (ELISA) (Innogenetics, Ghent, Belgium), which had an interassay precision of $3-6 \%$ and an intraassay precision of $2-5 \%$. Samples were tested pairwise per patient to reduce between-assay run variability (i.e., baseline and postbaseline samples in the same assay run and on the same microtiter ELISA plate). All biosamples were analyzed in a centralized location at the Janssen Alzheimer Immunotherapy Research \& Development Bioanalytical Development laboratory in South San Francisco, CA, USA. All prespecified endpoints are listed in Additional file 3.

\section{Statistical methods}

For the ApoE $\varepsilon 4$ carrier study, approximately 480 patients were to be randomized to bapineuzumab $0.5 \mathrm{mg} / \mathrm{kg}$ and 320 to placebo. This number of patients gave $90 \%$ power to detect a 2.21-point advantage for the bapineuzumab group over placebo on ADAS-Cog/11 
total score and a 5.39-point advantage on DAD total score at week 78. For the ApoE $\varepsilon 4$ noncarrier study, approximately 295 patients were to be randomized to each bapineuzumab dose group $(0.5$ and $1.0 \mathrm{mg} / \mathrm{kg})$ and 400 patients to placebo, giving $90 \%$ power to detect a 2.65-point advantage on ADAS-Cog/11 total score for a bapineuzumab dose over placebo and a 6.56-point advantage on DAD total score at week 78 . The standard deviations for the ADAS-Cog/11 total score and the DAD total score used in the power calculation were 9.3 and 23.0, respectively (based on previous studies). Planned substudy enrollment in the carrier substudies was 80 patients for the PiB-PET substudy, 300 for the CSF substudy, and 680 for the vMRI substudy. In the noncarrier study, planned enrollment was 90 patients for the PiB-PET substudy, 190 for the CSF substudy, and 550 for the vMRI substudy.

\section{Study populations}

Study populations included Safety: all randomized patients who received at least one infusion or portion of an infusion of study drug; Modified intention-to-treat (mITT): subjects in the Safety population who had a baseline assessment and at least one postbaseline assessment of ADAS-Cog/11 and DAD total scores; All PiB-PET, CSF, and vMRI: subjects in the Safety population who were enrolled in the specified substudy and had a valid baseline assessment and at least one postbaseline measurement; PiB-PET: patients in the All PiB-PET population who had a baseline $\mathrm{SUVr} \geq 1.35$, the threshold for amyloid positivity, and had at least one postbaseline measurement. A restricted maximum likelihoodbased mixed model for repeated measures (MMRM) was used to analyze the coprimary efficacy endpoints. Primary analysis was based on treatment difference using least squares means, with factor levels weighted according to overall baseline sample proportions. CSF biomarkers were analyzed using analysis of covariance, since week 71 was the only postbaseline assessment.

\section{Results}

\section{Patient disposition}

In the ApoE \&4 carrier study, 1099 patients were randomized and 1093 were treated (654 bapineuzumab $0.5 \mathrm{mg} / \mathrm{kg}, 439$ placebo) (Fig. 1). A total of 1081 patients were included in the mITT population (650 bapineuzumab, 431 placebo). Three hundred ninety-eight treated patients $(60.9 \%)$ in the bapineuzumab group and 285 $(64.9 \%)$ in the placebo group completed the study (60.5\% and $64.6 \%$ of randomized subjects, respectively) (Fig. 1). The most common reason for discontinuation was study termination by the sponsor ( $13.5 \%$ bapineuzumab, $14.8 \%$ placebo). Withdrawal due to adverse events (AEs) was higher for the bapineuzumab group $(9.0 \%)$ than for the placebo group (7.3 \%) (Fig. 1).
In the ApoE \&4 noncarrier study, 890 patients were randomized with 885 treated (267 bapineuzumab $0.5 \mathrm{mg} / \mathrm{kg}, 263$ bapineuzumab $1.0 \mathrm{mg} / \mathrm{kg}, 11$ bapineuzumab $2.0 \mathrm{mg} / \mathrm{kg}$, 344 placebo) (Fig. 1). The mITT population included 847 patients (255 bapineuzumab $0.5 \mathrm{mg} /$ $\mathrm{kg}, 253$ bapineuzumab $1.0 \mathrm{mg} / \mathrm{kg}, 11$ bapineuzumab $2.0 \mathrm{mg} / \mathrm{kg}, 328$ placebo). Patients in the $2.0 \mathrm{mg} / \mathrm{kg}$ group were not included in the primary efficacy analysis or safety analysis. Three hundred twenty-nine treated patients (37.2\%) completed the study (102 [38.2\%], 94 [35.7\%], 9 [81.8\%], and 124 [36.0\%] in the bapineuzumab $0.5 \mathrm{mg} / \mathrm{kg}, 1.0 \mathrm{mg} / \mathrm{kg}, 2.0 \mathrm{mg} / \mathrm{kg}$, and placebo groups, respectively). A total of 556 treated patients withdrew, with the most common reason being sponsor decision to terminate the study $(48.3 \%, 44.9 \%$, and $45.1 \%$ in the bapineuzumab $0.5 \mathrm{mg} / \mathrm{kg}, 1.0 \mathrm{mg} / \mathrm{kg}$, and placebo groups, respectively); withdrawal due to AEs was comparable across treatment groups (4.9\%, $4.9 \%$, and $5.5 \%$, respectively) (Fig. 1).

\section{Exposure}

In the carrier study safety population, the median duration of exposure was 1.49 years in both groups. All six infusions were administered to $57.4 \%$ of patients in the bapineuzumab $0.5 \mathrm{mg} / \mathrm{kg}$ group and $69.8 \%$ in the placebo group. In the noncarrier study safety population, the median duration of exposure was 1 year in all groups. All six infusions were administered to $41.6 \%$, $38.3 \%$, and $40.9 \%$ in the bapineuzumab $0.5 \mathrm{mg} / \mathrm{kg}$, bapineuzumab $1.0 \mathrm{mg} / \mathrm{kg}$, and placebo groups, respectively, in the mITT population (Table 1). Among the 11 patients in the $2.0 \mathrm{mg} / \mathrm{kg}$ group, the median duration of exposure was 1 year and all six infusions were received by $54.5 \%$ of patients in the mITT population.

\section{Demographics and baseline characteristics}

In both studies, mean age at study baseline was similar across treatment groups, and the majority of patients were white and female (Table 1). Approximately $17 \%$ were Asian, as both studies recruited patients from 36 centers in Japan. The mean duration of $\mathrm{AD}$ diagnosis ranged between 2.6 and 3.0 years, and $\geq 80 \%$ in each group were being treated with cholinesterase inhibitors and/or memantine. The distribution of randomization stratification factors was similar across groups; among carriers, the majority of patients (approximately $77 \%$ ) had a single ApoE $\varepsilon 4$ allele.

\section{Clinical efficacy}

In the ApoE \&4 carrier study, there was no statistically significant difference between the bapineuzumab $0.5 \mathrm{mg} / \mathrm{kg}$ and placebo groups for ADAS-Cog/11 $(p=0.979)$ or DAD $(p=0.973)$ (Fig. 2). Similarly, in noncarriers, no statistically significant difference was observed for ADAS-Cog/11 or 


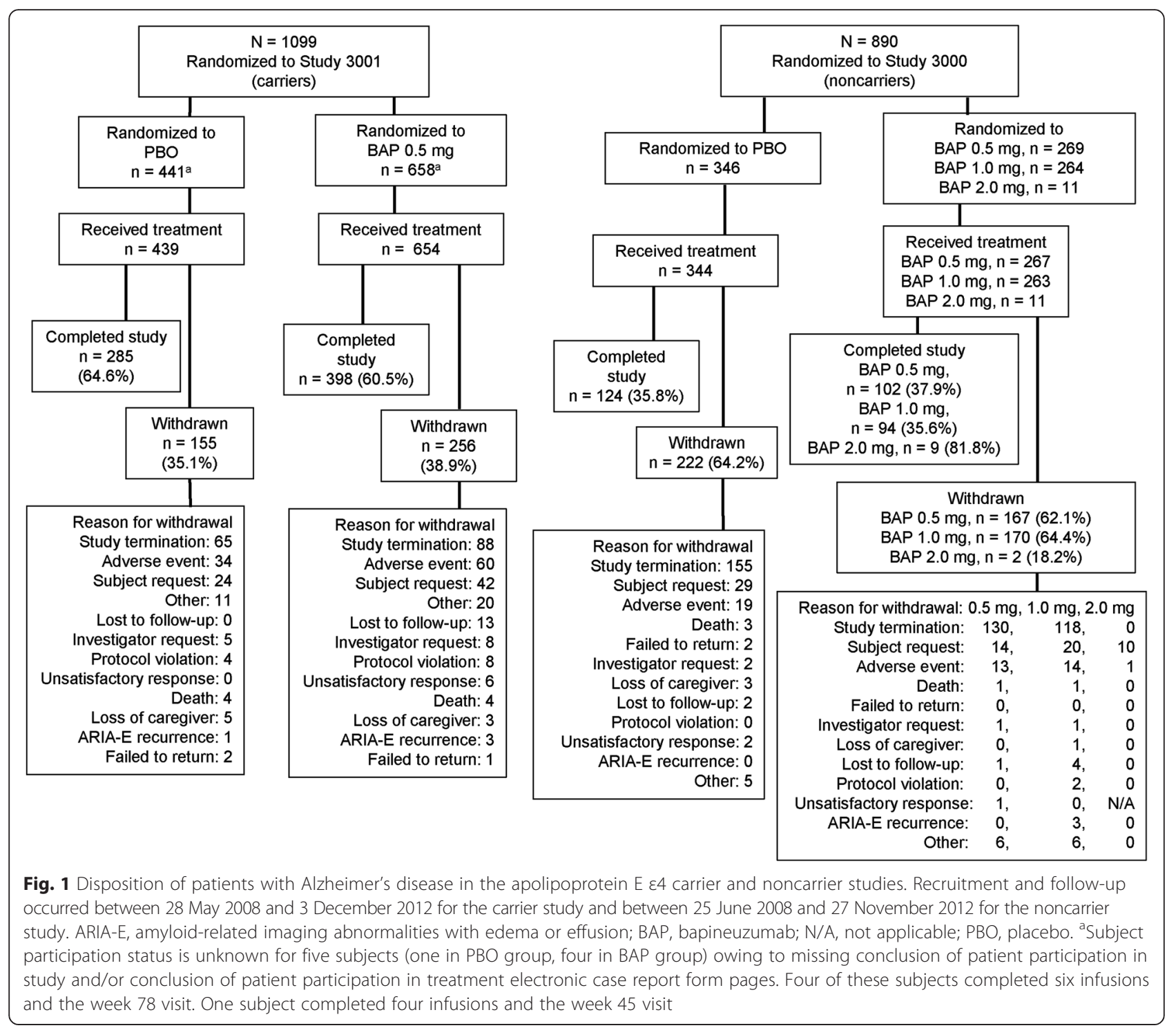

DAD between the bapineuzumab $0.5 \mathrm{mg} / \mathrm{kg}$ and placebo groups ( $p=0.057$ and $p=0.459$, respectively) or between the bapineuzumab $1.0 \mathrm{mg} / \mathrm{kg}$ and placebo groups $(p=$ 0.848 and $p=0.623$, respectively). An analysis of coprimary endpoints among completers yielded similar findings; no significant differences were observed for ADAS-Cog/11 or DAD in either study. For a subgroup analysis by disease severity and $A p o E \varepsilon 4$ carrier status, see Additional file 4: Table S1.

\section{Additional efficacy endpoints}

At week 78 , there were no significant differences between bapineuzumab and placebo in the NTB total Z-score, CDR-SOB score, or Dependence Scale total score in either study, with one exception: in the noncarrier study, there was a significant difference in favor of bapineuzumab between bapineuzumab $0.5 \mathrm{mg} / \mathrm{kg}$ and placebo on NTB total Z-score (difference 0.10, $p=0.047)$. All additional efficacy endpoint data are shown in Table 2.

\section{Biomarkers \\ PIB-PET}

Change from baseline to week 71 for PiB-PET GCA SUVr was not statistically significant versus placebo in either study (Fig. 3a). Fifty-seven patients were enrolled in the ApoE $\varepsilon 4$ carrier substudy and 39 in the noncarrier substudy. Twenty-three percent of patients did not meet the threshold of SUVr for the GCA region of interest $\geq 1.35$ at baseline on PiB-PET in noncarriers (vs $2 \%$ of carriers); all amyloid-negative patients were excluded from the PiB-PET population analysis. Baseline values of PiB-PET GCA SUVr (mean [standard deviation]) were similar between carriers: $2.2(0.29)$ in the 
Table 1 Patient demographics and baseline characteristics (modified intention-to-treat population)

\begin{tabular}{|c|c|c|c|c|c|}
\hline & \multicolumn{2}{|c|}{ ApoE $\varepsilon 4$ carrier study } & \multicolumn{3}{|c|}{ ApoE $\varepsilon 4$ noncarrier study } \\
\hline & Placebo $(n=431)$ & BAP $0.5(n=650)$ & Placebo $(n=328)$ & BAP $0.5(n=255)$ & BAP $1.0(n=253)$ \\
\hline Mean age, years & 70.2 & 70.9 & 69.7 & 71.1 & 70.7 \\
\hline Female, \% & 60.1 & 64.5 & 57.9 & 55.7 & 57.3 \\
\hline White, \% & 82.6 & 79.5 & 80.5 & 79.2 & 79.4 \\
\hline Asian, \% & 16.0 & 17.7 & 17.1 & 17.3 & 17.4 \\
\hline Black, \% & 0.7 & 0.8 & 0.6 & 0.8 & 2.0 \\
\hline Other, \% & 0.7 & 2.0 & 1.8 & 2.7 & 1.2 \\
\hline Mean duration of $A D$, years (SD) & $2.9(2.2)$ & $3.0(2.2)$ & $2.8(2.5)$ & $2.6(2.3)$ & $2.9(2.2)$ \\
\hline \multicolumn{6}{|l|}{ ApoE $\varepsilon 4$ allele status, $n(\%)$} \\
\hline Heterozygous & $334(77.5)$ & $500(76.9)$ & - & - & - \\
\hline Homozygous & $97(22.5)$ & $150(23.1)$ & - & - & - \\
\hline Using anti-AD medication at baseline, $n(\%)$ & $386(89.6)$ & $578(88.9)$ & $274(83.5)$ & $204(80.0)$ & $209(82.6)$ \\
\hline Mean MMSE score (SD) & $21.0(3.0)$ & $20.9(3.1)$ & $20.8(3.1)$ & $20.8(3.2)$ & $20.8(3.1)$ \\
\hline Mean years of formal education (SD) & $12.5(3.6)$ & $12.2(3.7)$ & $11.8(3.9)$ & $11.9(3.9)$ & $11.8(3.9)$ \\
\hline \multicolumn{6}{|l|}{ Substudy participation, n (\%) } \\
\hline $\mathrm{VMRI}+\mathrm{PET}$ or $\mathrm{VMRI}+\mathrm{PET}+\mathrm{CSF}$ & $47(10.9)$ & $64(9.8)$ & $34(10.4)$ & $27(10.6)$ & $21(8.3)$ \\
\hline CSF or CSF + VMRI & $104(24.1)$ & $160(24.6)$ & $67(20.4)$ & $44(17.3)$ & $53(20.9)$ \\
\hline vMRI only & $161(37.4)$ & $241(37.1)$ & $112(34.1)$ & $92(36.1)$ & $88(34.8)$ \\
\hline No substudy & $119(27.6)$ & $185(28.5)$ & $115(35.1)$ & $92(36.1)$ & $91(36.0)$ \\
\hline Mean ADAS-Cog/11 score (SD) & $22.6(8.9)$ & $23.2(8.9)$ & $22.9(10.2)$ & $23.2(10.0)$ & $23.5(9.3)$ \\
\hline Mean DAD score (SD) & 80.9 (18.7) & 79.9 (18.3) & $79.6(17.9)$ & $78.6(20.0)$ & $79.0(18.4)$ \\
\hline \multicolumn{6}{|l|}{ Infusions received, n (\%) } \\
\hline 1 & $13(3.0)$ & $34(5.2)$ & $40(12.2)$ & $22(8.6)$ & $29(11.5)$ \\
\hline 2 & $17(3.9)$ & $42(6.5)$ & $44(13.4)$ & $37(14.5)$ & $46(18.2)$ \\
\hline 3 & $29(6.7)$ & $57(8.8)$ & $36(11.0)$ & $31(12.2)$ & $25(9.9)$ \\
\hline 4 & $28(6.5)$ & $46(7.1)$ & $33(10.1)$ & $24(9.4)$ & $26(10.3)$ \\
\hline 5 & $43(10.0)$ & $98(15.1)$ & $40(12.2)$ & $35(13.7)$ & $30(11.9)$ \\
\hline 6 & $301(69.8)$ & $373(57.4)$ & $134(40.9)^{a}$ & $106(41.6)$ & $97(38.3)$ \\
\hline
\end{tabular}

$A D$ Alzheimer's disease, ADAS-Cog/11 11-item Alzheimer's Disease Assessment Scale-Cognitive subscale, ApoE apolipoprotein E, BAP bapineuzumab, CSF cerebrospinal fluid, DAD Disability Assessment for Dementia, MMSE Mini Mental State Examination, $P E T$ positron emission tomography, vMRI volumetric magnetic resonance imaging ${ }^{a}$ One patient in the placebo group received seven infusions

placebo group and $2.2(0.31)$ in the bapineuzumab group, and noncarriers: $2.1(0.21)$ in the placebo group and $2.1(0.24)$ in the pooled bapineuzumab group. A plot of the baseline values for all patients screened for the PiBPET substudy (Fig. 4) shows the separation between noncarriers who were classified as amyloid-negative and those who were classified as amyloid-positive. A total of 14 noncarriers and 27 carriers received the week 71 PET imaging assessment. To estimate the effect of premature study termination on statistical power, $p$ values were calculated assuming that the data trend continued with full enrollment in each study. The $p$ value in the carrier study was estimated to be 0.085 comparing bapineuzumab with placebo, and the $p$ value was estimated to be 0.438 in the noncarrier study comparing pooled bapineuzumab with placebo.

\section{CSF $p$-tau}

The CSF carrier substudy enrolled 138 patients and the noncarrier substudy enrolled 76. Nearly all had a baseline and week 71 assessment. No significant differences were observed in CSF p-tau, although in noncarriers the difference showed a trend $(p=0.085)$ favoring the bapineuzumab pooled group (Fig. $3 \mathrm{~b}$ ).

\section{vMRI}

The vMRI carrier substudy enrolled 540 patients and the noncarrier substudy enrolled 401. No significant treatment difference was observed in annualized rate (milliliters per year) of whole-brain volume loss assessed by BBSI in either study (Fig. 3c). In all groups, the annual estimated decrease in whole-brain volume was approximately $18 \mathrm{ml} /$ year across all treatment 


\section{A ADAS-Cog/11}
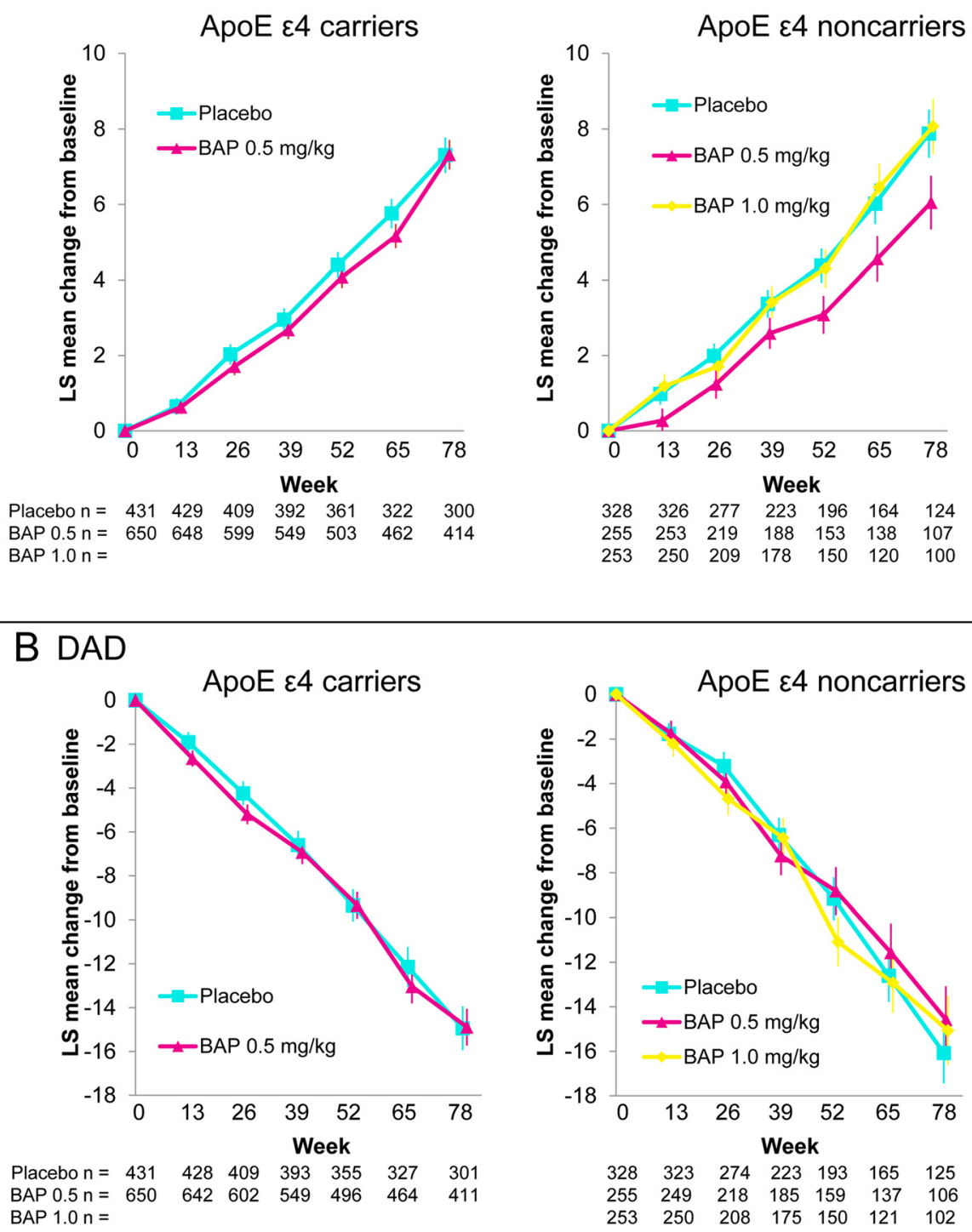

Fig. 2 Primary efficacy outcome analysis: change from baseline to week 78. a ADAS-Cog/11: total score range is 0 (least impairment) to 70 (most impairment). A positive change from baseline indicates worsening cognitive impairment. b DAD: total score range is 0 to 100, with higher scores indicating better function. A negative change from baseline indicates worsening function. Data shown are least squares means with standard error of the mean. ADAS-Cog/11, 11-item Alzheimer's Disease Assessment Scale-Cognitive subscale; ApoE, apolipoprotein E; BAP, bapineuzumab; DAD, Disability Assessment for Dementia; LS, least squares

groups at the first follow-up assessment (week 19) as well as at subsequent assessments.

\section{Pharmacodynamics}

Significant increases in plasma $\mathrm{A} \beta$ levels with bapineuzumab from baseline to week 71 were observed in both carrier (bapineuzumab-placebo difference $827.29 \mathrm{pg} / \mathrm{ml}$, $p<0.001$ ) and noncarrier (pooled bapineuzumabplacebo difference $941.86 \mathrm{pg} / \mathrm{ml}, p<0.001)$ studies (Fig. 3d).

\section{Safety}

Treatment-emergent adverse events (TEAEs) with $>3 \%$ difference in incidence between groups were ARIA-E and cerebral microhemorrhage (more with bapineuzumab) and anxiety and headache (more with placebo). For ApoE \&4 carriers, TEAEs considered treatmentrelated were reported in $34.6 \%$ of patients in the bapineuzumab group and $22.1 \%$ in the placebo group. In the ApoE \&4 noncarrier study, the incidence of TEAEs considered treatment-related was similar in the bapineuzumab $0.5 \mathrm{mg} / \mathrm{kg}$ and placebo groups $(18.4 \%$ and 
Table 2 Secondary and exploratory efficacy analyses: change from baseline to week 78

\begin{tabular}{|c|c|c|c|c|c|}
\hline & \multicolumn{2}{|c|}{ ApoE $\varepsilon 4$ carrier study } & \multicolumn{3}{|c|}{ ApoE $\varepsilon 4$ noncarrier study } \\
\hline & $\mathrm{PBO}(n=431)$ & BAP $0.5(n=650)$ & $\mathrm{PBO}(n=328)$ & BAP $0.5(n=255)$ & BAP $1.0(n=253)$ \\
\hline \multicolumn{6}{|l|}{ CDR-SOB total score ${ }^{a}$} \\
\hline Number of subjects & 310 & 427 & 144 & 115 & 110 \\
\hline Mean change (SD) & $2.4(2.8)$ & $2.3(2.9)$ & $2.5(2.8)$ & $2.2(2.8)$ & $2.2(2.6)$ \\
\hline \multicolumn{6}{|l|}{ MMRM analysis } \\
\hline LS mean change (SE) & $2.59(0.16)$ & $2.44(0.13)$ & $2.59(0.20)$ & $2.23(0.23)$ & $2.41(0.23)$ \\
\hline Difference vs PBO & & -0.15 & & -0.36 & -0.18 \\
\hline$p$ Value & & 0.448 & & 0.238 & 0.564 \\
\hline \multicolumn{6}{|l|}{ DS total score ${ }^{b}$} \\
\hline Number of subjects & 316 & 437 & 145 & 121 & 112 \\
\hline Mean change (SD) & $1.2(2.2)$ & $1.2(2.3)$ & $1.4(2.5)$ & $1.3(2.0)$ & $1.1(2.5)$ \\
\hline \multicolumn{6}{|l|}{ MMRM analysis } \\
\hline LS mean change (SE) & $1.33(0.1)$ & $1.22(0.1)$ & $1.45(0.17)$ & $1.29(0.19)$ & $1.16(0.19)$ \\
\hline Difference vs PBO & & -0.11 & & -0.16 & -0.29 \\
\hline$p$ Value & & 0.462 & & 0.516 & 0.257 \\
\hline \multicolumn{6}{|l|}{ NTB total Z-score ${ }^{c}$} \\
\hline Number of subjects & 296 & 403 & 120 & 105 & 96 \\
\hline Mean change (SD) & $0.0(0.5)$ & $0.0(0.6)$ & $-0.1(0.5)$ & $0(0.5)$ & $0(0.4)$ \\
\hline \multicolumn{6}{|l|}{ MMRM analysis } \\
\hline LS mean change (SE) & $-0.11(0.03)$ & $-0.10(0.02)$ & $-0.09(0.04)$ & $0.02(0.04)$ & $-0.12(0.04)$ \\
\hline Difference vs PBO & & 0.01 & & 0.10 & -0.03 \\
\hline$p$ Value & & 0.889 & & 0.047 & 0.541 \\
\hline
\end{tabular}

ApoE apolipoprotein E, BAP bapineuzumab, CDR-SOB Clinical Dementia Rating-Sum of Boxes, DS Dependence Scale, $L S$ least squares, MMRM mixed model for repeated measures, NTB Neuropsychological Test Battery, $P B O$ placebo

${ }^{a} \mathrm{CDR}$-SOB total score range is 0 (least impairment) to 18 (most impairment); a negative change from baseline indicates improvement

${ }^{b} \mathrm{DS}$ total score range is $0-15$, with higher scores indicating worse impairment; a negative change from baseline indicates improvement

'Positive change indicates improvement in NTB total Z-score

$18.3 \%$ of patients, respectively), but it was higher in the bapineuzumab $1.0 \mathrm{mg} / \mathrm{kg}$ group (30.0\%). The incidences of ARIA-E for ApoE \&4 carriers were $16.7 \%$ for the bapineuzumab $0.5 \mathrm{mg} / \mathrm{kg}$ group and $2.1 \%$ for the placebo group. Discontinuation due to ARIA-E was higher in the bapineuzumab group ( $2.9 \%$ vs $0.5 \%)$. All prespecified events of clinical importance other than ARIA-E and hypersensitivity reactions were reported in $\leq 1.2 \%$ in both treatment groups (Table 3 ). Seizure, deep vein thrombosis/pulmonary embolism, and intracranial hemorrhage were numerically more frequent, and hypersensitivity reaction was less frequent, in the bapineuzumab group than in the placebo group. For ApoE $\varepsilon 4$ carriers, the incidences of intraparenchymal hemorrhage were $0.46 \%$ and $0.76 \%$ in the placebo and bapineuzumab groups, respectively (Table 3 ).

In noncarriers, incidences of ARIA-E were $4.9 \%$ in the bapineuzumab $0.5 \mathrm{mg} / \mathrm{kg}$ group, $11.8 \%$ in the bapineuzumab $1.0 \mathrm{mg} / \mathrm{kg}$ group, and $0.6 \%$ in the placebo group (Table 3). Discontinuation due to ARIA-E was highest in the bapineuzumab $1.0 \mathrm{mg} / \mathrm{kg}$ group compared with the bapineuzumab $0.5 \mathrm{mg} / \mathrm{kg}$ and placebo groups (3.0\% vs $1.0 \%$ and $0.6 \%$, respectively). All prespecified AEs other than ARIA-E occurred in $\leq 2.0 \%$ in all three groups. Incidences of intracranial hemorrhage and seizure/convulsion were higher in the placebo group than in either bapineuzumab group. There was one case of deep vein thrombosis/pulmonary embolism in each of the bapineuzumab groups and no cases in the placebo group; no cases of intraparenchymal hemorrhage occurred in the noncarrier study.

\section{Discussion}

The presently reported studies (3000/3001) are the last of four phase 3 trials performed to evaluate bapineuzumab immunotherapy. As in the first two U.S. trials (Studies 301 and 302), no significant differences were found in the coprimary cognitive or functional endpoints in this more global demographic [3]. Early termination of the $3000 / 3001$ studies could have contributed to the 


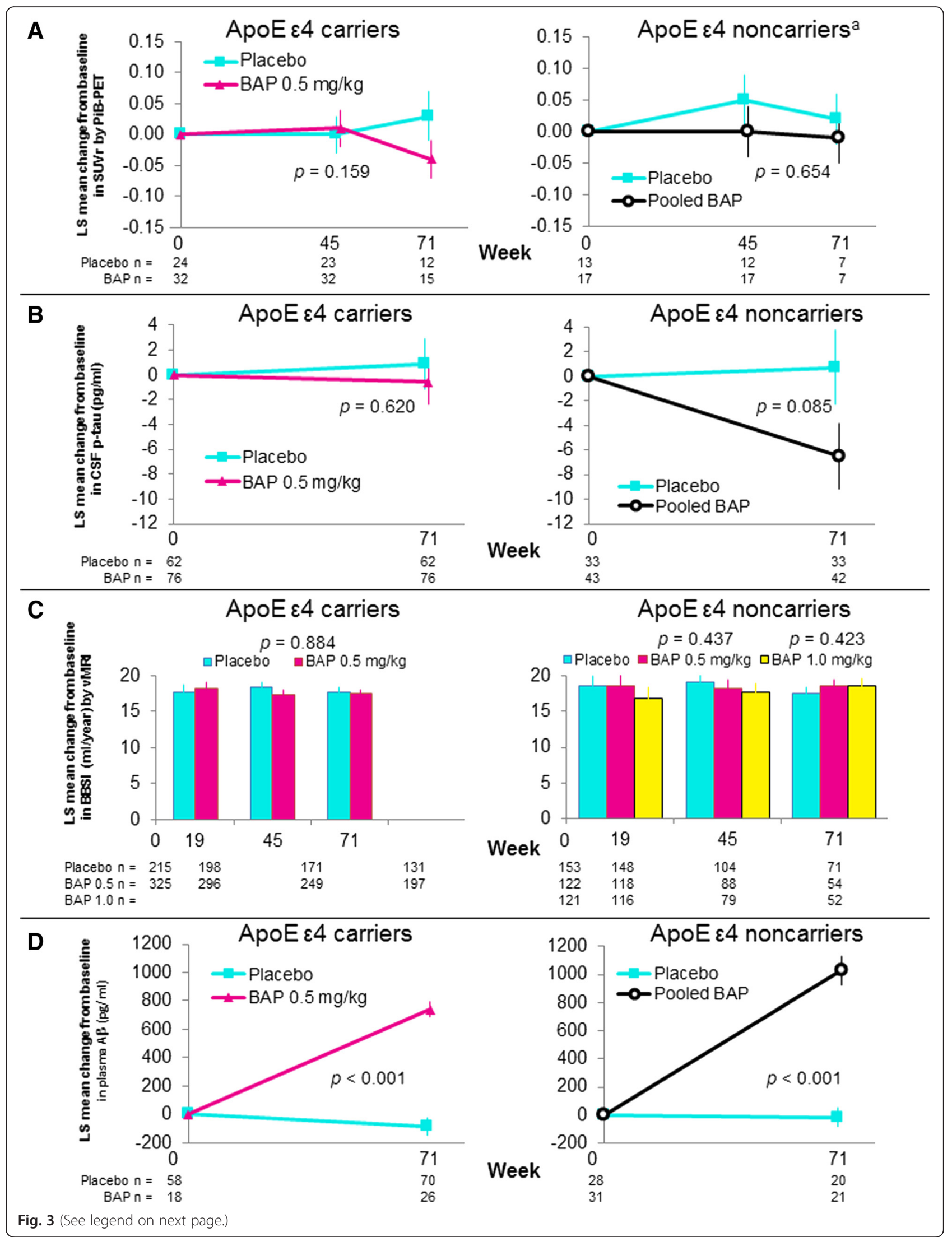


(See figure on previous page.)

Fig. 3 Analysis of biomarkers and plasma $A B$ : change from baseline to week 71. Data shown are least squares means with standard error of the mean. a PiB-PET analysis (b) CSF p-tau analysis (c) Volumetric analysis: An increase in BBSI on vMRI indicates a decrease in brain volume. $\mathbf{d}$ Plasma $A \beta$ analysis. $A \beta$, amyloid $\beta$; ApoE, apolipoprotein E; BAP, bapineuzumab; BBSI, brain boundary shift integral; CSF, cerebrospinal fluid; LS, least squares; PiB-PET, ${ }^{11} \mathrm{C}$-Pittsburgh compound B positron emission tomography; p-tau, phosphorylated tau protein; SUVr, standardized uptake value ratio; VMRI, volumetric magnetic resonance imaging. ${ }^{\text {E}}$ Excludes nine patients who were PiB-PET-negative for $A \beta$ at baseline

inability to detect a treatment effect; however, enrollment in the carrier (3001) study was complete (only the PET substudy was still recruiting), MMRM analysis was used, the treatment and placebo values were similar, and the negative results in the mITT and completer populations were consistent with studies $301 / 302$ collectively, suggesting that the lack of clinical effect seen in these two studies was not due to early termination. There was no evidence of a clinical dose effect in the noncarrier study between the $0.5 \mathrm{mg} / \mathrm{kg}$ and $1.0 \mathrm{mg} / \mathrm{kg}$ doses. The small number of patients in the $2.0 \mathrm{mg} / \mathrm{kg}$ dose group (11 patients, 9 completers) and the reassignment of these patients to $1.0 \mathrm{mg} / \mathrm{kg}$ did not permit evaluation of the effects of higher doses.

The need for earlier intervention with amyloidlowering therapy has been hypothesized as a potential reason for lack of clinical efficacy observed in recent phase 3 studies of bapineuzumab and of solanezumab, another anti-A $\beta$-targeted monoclonal antibody $[2,3,8,9]$. Also, a statistically significant benefit of bapineuzumab was reported in Study 301 in a mild AD subgroup (MMSE >19) of ApoE $\varepsilon 4$ noncarriers on the functional but not the cognitive measure [3]. On the basis of these findings, phase 3 studies in patients with mild $\mathrm{AD}$ and prodromal $\mathrm{AD}$ were initiated with solanezumab and another anti-A $\beta$-targeted monoclonal antibody, gantenerumab [10]. Twenty-three percent of noncarriers and two percent of carriers who had baseline PiB-PET scans met the clinical criteria for
AD dementia but did not meet the PiB-PET threshold for amyloid positivity at baseline. These patients with "suspected nonamyloid pathology" may have had some other form of dementia that could not be differentiated from $\mathrm{AD}$ on the basis of clinical inclusion criteria. Patients with low levels of amyloid would not be expected to benefit from an antiamyloid therapy. Inclusion of subthreshold amyloid patients was also a factor in the 301/302 bapineuzumab PiB-PET substudies (36 \% and $6.5 \%$ of those with baseline scans in the noncarrier and carrier studies, respectively) [3]. The proportion of patients not meeting the amyloid threshold was lower in the 3000/3001 studies than in the 301/302 studies using the same threshold value, suggesting some differences in enrollment between the two sets of studies. The choice of amyloid threshold was based on the consensus of experts consulted at the time the studies were initiated, but the plot of baseline values shows a clear separation between positive and negative populations that would be robust to a range of selected thresholds. The amyloid-negative patients were included in the analyses of outcomes other than SUVr; however, it is unlikely that exclusion of the ten patients who were amyloid-negative would affect the overall study outcome. Since brain amyloid positivity was not an inclusion criterion, it is not possible to evaluate its impact on the observed cognitive and functional outcomes. Collectively, the findings from these studies indicate that amyloid assessment at screening is essential and that meeting an

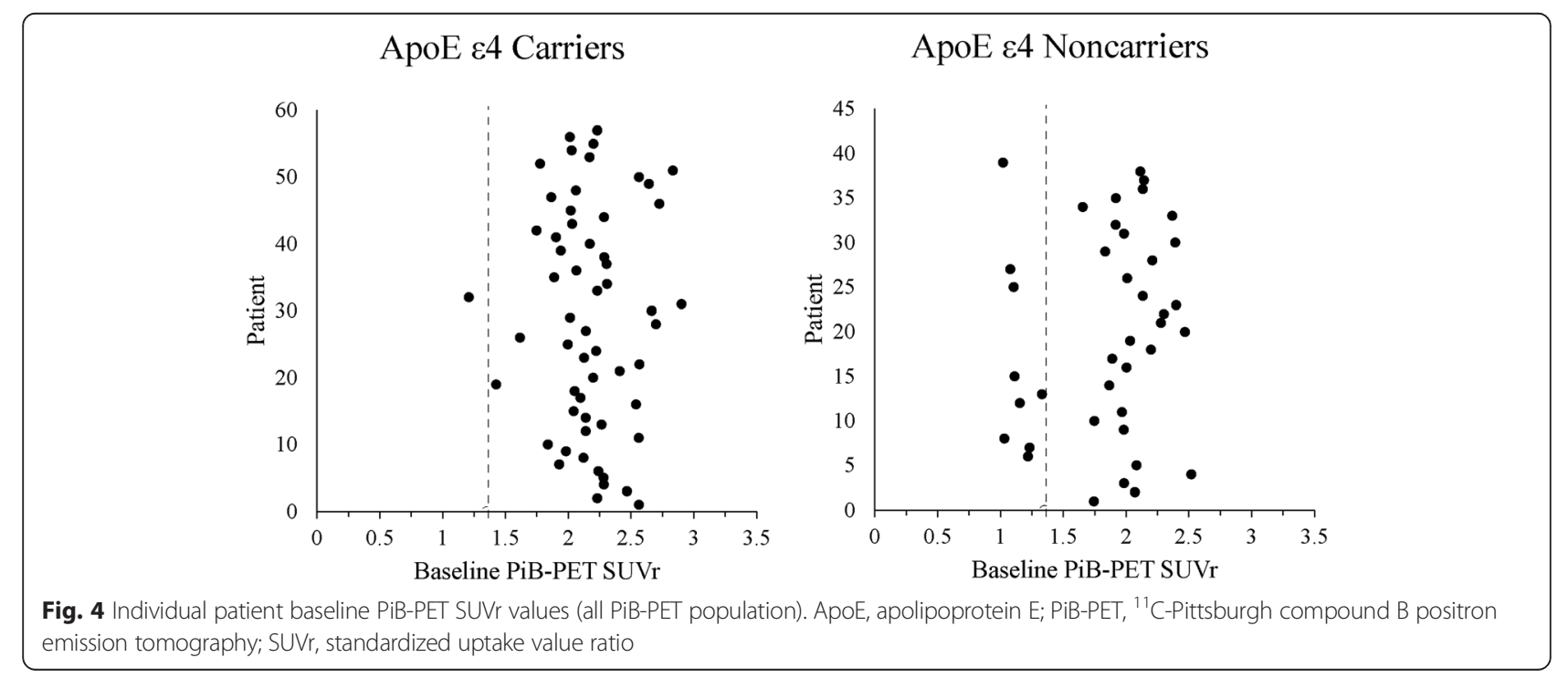


Table 3 Prespecified events of clinical importance-incidence proportion (95\% Cl)

\begin{tabular}{|c|c|c|c|c|c|}
\hline \multirow[t]{3}{*}{ Event, n, \% (95\% Cl) } & \multicolumn{2}{|c|}{ ApoE ع4 carrier study } & \multicolumn{3}{|c|}{ ApoE $\varepsilon 4$ noncarrier study } \\
\hline & Placebo & BAP 0.5 & Placebo & BAP 0.5 & BAP 1.0 \\
\hline & $(n=439)$ & $(n=654)$ & $(n=344)$ & $(n=267)$ & $(n=263)$ \\
\hline \multirow[t]{3}{*}{ ARIA-E } & 9 & 109 & 2 & 13 & 31 \\
\hline & 2.05 & 16.67 & 0.58 & 4.87 & 11.79 \\
\hline & $(0.94,3.86)$ & $(13.89,19.75)$ & $(0.07,2.08)$ & $(2.62,8.18)$ & $(8.15,16.3)$ \\
\hline \multirow[t]{3}{*}{ Intracranial hemorrhage } & 5 & 6 & 7 & 2 & 1 \\
\hline & 1.14 & 0.92 & 2.03 & 0.75 & 0.38 \\
\hline & $(0.37,2.64)$ & $(0.34,1.99)$ & $(0.82,4.15)$ & $(0.09,2.68)$ & $(0.01,2.10)$ \\
\hline \multirow[t]{3}{*}{ Seizures/convulsions } & 1 & 7 & 3 & 1 & 0 \\
\hline & 0.23 & 1.07 & 0.87 & 0.37 & 0.00 \\
\hline & $(0.01,1.26)$ & $(0.43,2.19)$ & $(0.18,2.53)$ & $(0.01,2.07)$ & $(0.00,1.39)$ \\
\hline \multirow[t]{3}{*}{ DVT/PE } & 2 & 8 & 0 & 1 & 1 \\
\hline & 0.46 & 1.22 & 0.00 & 0.37 & 0.38 \\
\hline & $(0.06,1.64)$ & $(0.53,2.40)$ & $(0.00,1.07)$ & $(0.01,2.07)$ & $(0.01,2.10)$ \\
\hline \multirow[t]{3}{*}{ Hypersensitivity reactions } & 19 & 18 & 6 & 5 & 4 \\
\hline & 4.33 & 2.75 & 1.74 & 1.87 & 1.52 \\
\hline & $(2.63,6.68)$ & $(1.64,4.32)$ & $(0.64,3.76)$ & $(0.61,4.32)$ & $(0.42,3.85)$ \\
\hline \multirow[t]{3}{*}{ Intraparenchymal hemorrhage } & 2 & 5 & 0 & 0 & 0 \\
\hline & 0.46 & 0.76 & 0.00 & 0.00 & 0.00 \\
\hline & $(0.06,1.64)$ & $(0.25,1.78)$ & $(0.00,1.07)$ & $(0.00,1.37)$ & $(0.00,1.39)$ \\
\hline
\end{tabular}

ApoE apolipoprotein E, ARIA-E amyloid-related imaging abnormalities, edema/effusion, BAP bapineuzumab, DVT/PE deep vein thrombosis/pulmonary embolism

agreed amyloid threshold should be an inclusion criterion for future antiamyloid therapy trials.

The 3000/3001 bapineuzumab studies did not fully replicate the PiB-PET or CSF biomarker findings from Studies 301/302. In Studies 3000/3001, no significant treatment differences were seen in amyloid burden on PiB-PET or CSF p-tau (but trends were in the expected directions) (Fig. 3b), which appeared to be related to stability of the placebo groups over time. This finding differs from the results of Study 302 in ApoE \&4 carriers, in which SUVr on PiB-PET continued to increase in the placebo group with no change in the bapineuzumab group, suggesting prevention of $A \beta$ accumulation. Significant decreases in amyloid load on PiB-PET with bapineuzumab were also reported from a phase 2 study [6]. However, Study 301 in noncarriers showed no change in SUVr in the placebo group and no significant difference at week 71 between placebo and the bapineuzumab $0.5 \mathrm{mg} / \mathrm{kg}$ and $1.0 \mathrm{mg} / \mathrm{kg}$ groups [3]. Mean amyloid load at baseline in amyloid-positive patients was similar between carriers and noncarriers in all four studies and between the 301/302 and 3000/3001 studies. A possible reason for lack of significance of the PiB-PET results in Studies 3000/3001 is the small number of subjects who completed each PiB-PET substudy, owing to early study termination. Only 27 patients in the current carrier study and 14 in the noncarrier study (approximately half in the bapineuzumab and half in the placebo group in each study) had an assessment at week 71 , in contrast to 115 patients (75 bapineuzumab, 40 placebo) in the 302 carrier study and 39 patients (24 bapineuzumab, 15 placebo) in the 301 noncarrier study [3]. However, the projected $p$ values for the PET substudies assuming full enrollment with the observed data trends suggest that full enrollment would not have changed the study conclusions. The cerebellar gray matter was used as reference region in the PET analyses, opening up another possibility that the use of the pons as reference region might have reduced the noise and thereby improved sensitivity to detect changes [11].

In Studies 3000/3001, there was no effect of treatment on CSF p-tau, although there was a trend for significant reduction in the pooled bapineuzumab group in noncarriers. This finding differs from the results of the 301/302 studies. In ApoE $\varepsilon 4$ noncarriers in the latter studies, there was no effect on CSF p-tau concentrations in the pooled bapineuzumab groups (the prespecified analysis), but exploratory analyses showed a significant reduction in CSF p-tau with bapineuzumab $1.0 \mathrm{mg} / \mathrm{kg}$ [3]. As was the case for the PiB-PET analysis of SUVr, the results for CSF p-tau in the current studies were likely affected by the small number of patients who completed the assessments. Compared with the 301/302 studies, the number of patients in the bapineuzumab groups was less by almost 
half. vMRI assessments showed no significant effect of bapineuzumab treatment in any of the four studies, and rates of whole-brain volume loss were similar [3].

Plasma $A \beta_{x-40}$ levels increased significantly in the bapineuzumab groups but not in the placebo group in the 3000/3001 studies, a result expected from anti-A $\beta$ antibody infusion observed in other studies and an indicator of peripheral target engagement $[2,9]$.

Infusions of bapineuzumab 0.5 and $1.0 \mathrm{mg} / \mathrm{kg}$ every 13 weeks were generally well-tolerated, and the safety profile was consistent with that reported in previous studies. No new or unexpected safety findings were observed $[3,7]$. ARIA-E were confirmed as dose-dependent TEAEs associated with bapineuzumab; these events increased with dose and ApoE $\varepsilon 4$ allele number and led to discontinuation of the $2.0 \mathrm{mg} / \mathrm{kg}$ dose in all noncarrier studies [3]. Rates of ARIA-E were three to four times higher in the placebo group of ApoE $\varepsilon 4$ carriers than among noncarriers in the current studies, which differs from what was reported in the previous studies. This difference may have been due to increased detection because of the central image-reading of every MRI scan, suggesting a need for more intensive radiologist training to detect ARIA-E, particularly in clinical trials. Further research is needed to identify risk factors for ARIA-E and their long-term clinical course [12]. There is growing evidence that ARIA is related to amyloid clearance from the brain [12], which could eventually be tested by comparing PET and MRI images from the four completed bapineuzumab studies.

\section{Study limitations}

Early termination is the major limitation of both studies, as both were discontinued earlier than expected and before enrollment was complete in the noncarrier study. Premature termination led to smaller-than-expected sample sizes, particularly in the PiB-PET and CSF p-tau substudies. In addition, assuming that the PET substudy population is representative of the entire study population, a relatively high proportion of ApoE $\varepsilon 4$ noncarriers did not meet a preestablished amyloid threshold and therefore lacked the drug target.

\section{Conclusions}

Despite early termination, these studies in a more global population demographic confirm the overall negative clinical findings of the first phase 3 trials of bapineuzumab in mild to moderate AD. Some biomarker results of the $301 / 302$ studies were not confirmed in the present studies, although small sample sizes limit their interpretation. In the future, a pooled analysis of the data from the four studies may provide more clarity on the effects of bapineuzumab on biomarkers of $\mathrm{AD}$. In the PiB-PET substudy, the percentage of ApoE $\varepsilon 4$ noncarriers with amyloid below the GCA threshold SUVr of 1.35 is lower in this study than in
Study 301 ( $23 \%$ vs $36 \%$ ). Assuming that the percentage is similar in the entire study population, it is still high and highlights the potential importance of meeting a preestablished amyloid threshold as an inclusion criterion when conducting future studies of this type. Preliminary studies to determine adequate blood-brain barrier penetration of therapeutic antibodies at planned study doses would also help answer questions about central target engagement. Large studies in patients with prodromal AD who have sufficient brain amyloid to confirm the diagnosis, with extended follow-up to ensure significant change from baseline in the placebo group, will likely be needed to ultimately determine the viability of an immunotherapeutic approach to $\mathrm{AD}$ treatment.

\section{Ethical approval and consent to participate}

These studies were approved by the institutional review board or independent ethics committee at each site, and each patient (or the patient's legal representative) and patient caregiver provided written informed consent before any screening procedures were performed. A complete list of all ethical bodies that approved the ApoE $\varepsilon 4$ carrier and ApoE $\varepsilon 4$ noncarrier studies are listed in Additional file 5 and Additional file 6, respectively.

\section{Availability of supporting data}

Requests for access to the original data should be addressed to the study sponsors.

\section{Additional files}

\section{Additional file 1: Coinvestigators. Complete list of study investigators.} (DOCX 46 kb)

Additional file 2: Inclusion and exclusion criteria. Inclusion and exclusion criteria, similar to those used in the 301/302 studies. (DOCX 18 kb)

Additional file 3: Prespecified outcomes in apolipoprotein E $\varepsilon 4$ carrier and noncarrier trials. All prespecified endpoints are listed. (DOCX $18 \mathrm{~kb}$ )

Additional file 4: Table S1. Change from baseline to week 78 by MMSE score at baseline (ApoE \&4 carriers and noncarriers). Table showing analysis of ADAS-Cog/11 and DAD scores by disease severity. (DOCX $14 \mathrm{~kb}$ )

Additional file 5: List of independent ethics committees or institutional review boards. Complete list of independent ethics committees for all sites that screened subjects for the ApoE $\varepsilon 4$ carrier study. (PDF $134 \mathrm{~kb}$ )

Additional file 6: List of independent ethics committees or institutional review boards. Complete list of independent ethics committees for all sites that screened subjects for the ApoE $\varepsilon 4$ noncarrier study. (PDF 339 kb)

\section{Abbreviations}

A $\beta$ : amyloid $\beta$; AD: Alzheimer's disease; ADAS-Cog/11: 11-item Alzheimer's Disease Assessment Scale-Cognitive subscale; AE: adverse event;

ApoE: apolipoprotein E; ARIA-E: amyloid-related imaging abnormalities with edema or effusion; BAP: bapineuzumab; BBSI: brain boundary shift integral; CDR-SOB: Clinical Dementia Rating-Sum of Boxes; CSF: cerebrospinal fluid; DAD: Disability Assessment for Dementia; DS: Dependence Scale; DVT/ PE: deep vein thrombosis/pulmonary embolism; ELISA: enzyme-linked immunosorbent assay; GCA: global cortical average; IV: intravenous; LS: least squares; mITT: modified intention to treat; MMRM: mixed model for repeated 
measures; MMSE: Mini Mental State Examination; MRI: magnetic resonance imaging; NTB: Neuropsychological Test Battery; PBO: placebo; PET: positron emission tomography; PiB: ${ }^{11} \mathrm{C}$-Pittsburgh compound B; p-tau: phosphorylated tau; SUVr: standardized uptake value ratio; TEAE: treatment-emergent adverse event; vMRI: volumetric magnetic resonance imaging.

\section{Competing interests}

Dr. Vandenberghe (RV) discloses that a clinical trial agreement for the 3000 and 3001 studies was in place between Pfizer Inc. and University Hospitals Leuven (principal investigator, RV).

Dr. Rinne is a member of the Teva Finland Oy scientific advisory board and serves as a neurology consultant for Clinical Research Services Turku (CRST) Ltd. As a neurology consultant for CRST Ltd, he is involved in contract research with AC Immune, Merck, Otsuka Pharmaceutical Co., Roche, and TauRx Therapeutics. He has not received personal compensation from these organizations

Dr. Boada has consulted for Araclon Biotech, Avid Radiopharmaceuticals, Bayer, Elan, Grifols, Janssen/Pfizer, Eli Lilly and Co., Neuroptix, Nutricia, Roche, Sanofi, and Servier Laboratories. She has received fees for lectures as well as funds for research from Araclon Biotech, Esteve, Grifols, Janssen, Novartis, Nutricia, Piramal, Pfizer, Wyeth, Roche, and Servier Laboratories. She has not received personal compensation from these organizations.

Dr. Katayama declares no competing interests.

Dr. Scheltens has served as a consultant to Pfizer, Novartis, Boehringer, Takeda, and Nutricia Research and has received research support from Merck and GE Healthcare. He has not received personal compensation from these organizations.

Dr. Vellas has served as a scientific board member for AstraZeneca, Eisai Co., Elan, Exhonit, GlaxoSmithKline, Eli Lilly and Co., Medivation, Meso Scale Discovery (MSD), Nestlé, Nutricia, Pfizer, Pierre Fabre Laboratories, Roche, Sanofi, Servier Laboratories, TauRx Therapeutics, and Wyeth. He has received research grants from AbbVie, AFFiRiS, Avid Radiopharmaceuticals, BristolMyers Squibb, Eisai Co., Elan, EnVivo Pharmaceuticals, Exhonit, Genentech, GlaxoSmithKline, Fondation IPSEN, Eli Lilly and Co., Medivation, MSD, Nutricia, Otsuka Pharmaceutical Co., Pharnext, Pfizer, Pierre Fabre Laboratories, Regeneron Pharmaceuticals, Roche, Sanofi, Servier Laboratories, TauRx Therapeutics, and Wyeth.

Dr. Tuchman has received honoraria and research funding from Pfizer, Janssen, Merck, AstraZeneca, and Amgen.

Dr. Gass declares no competing interests.

Dr. Fiebach declares no competing interests.

Dr. Hill is an employee of IXICO plc and holds stock and share options in IXICO plc. Through IXICO, he has provided services to Pfizer, Janssen Alzheimer Immunotherapy Research \& Development, Bristol-Myers Squibb, Eli Lilly and Co., Teva, Roche, Medlmmune, GlaxoSmithKline, Kyowa Hakko Kirin Co., Green Valley, Eisai Co., VirtualScopics, and ENKAM Pharmaceuticals. Dr. Hill holds non-study-related patents in image data management and image analysis. Also, through IXICO, he has received research grant support from the European grant sources Innovative Medicines Initiative and FP7, also including in the United Kingdom the Technology Strategy Board and the Medical Research Council.

Dr. Lobello is an employee of Pfizer Inc. and an owner of Pfizer Inc. stock. Dr. Li is an employee of Pfizer Inc. and an owner of Pfizer Inc. stock. Dr. McRae is an employee of Pfizer Inc. and an owner of Pfizer Inc. stock. Dr. Lucas is an employee of Pfizer Inc. and an owner of Pfizer Inc. stock. Dr. Evans is an employee of Pfizer Inc. and an owner of Pfizer Inc. stock. Dr. Booth is an employee of Pfizer Inc. and an owner of Pfizer Inc. stock. Mr. Luscan is an employee of Pfizer Inc. and an owner of Pfizer Inc. stock. Dr. Wyman was an employee of Pfizer Inc. at the time these studies were conducted and is an owner of Pfizer Inc. stock.

Dr. Hua is an employee of Pfizer Inc. and an owner of Pfizer Inc. stock. Dr. Yang is an employee of Pfizer Inc.

Dr. Brashear is an employee of Janssen Alzheimer Immunotherapy Research \& Development.

Dr. Black was an employee of Pfizer Inc. at the time these studies were conducted and is an owner of Pfizer Inc. stock.

All authors declare that they have no nonfinancial competing interests.

\section{Authors' contributions}

RV contributed to collection of data; provided technical assistance; supervised personnel; contributed drugs, reagents, equipment, or participants; and contributed to drafting and/or revision of the manuscript. JOR contributed to study conduct, collection of data, interpretation of the data, and drafting and/or revision of the manuscript. MB contributed to study conduct, interpretation of the data, and drafting and/or revision of the manuscript. SK contributed to study conduct, interpretation of the data, and drafting and/or revision of the manuscript. PS contributed to data acquisition, supervised patient recruitment and enrollment, and edited and approved the manuscript. BV contributed to the acquisition, analysis, and interpretation of data as well as drafting and/or revision of the manuscript. MT contributed to the design of the studies; acquisition, analysis, and interpretation of the data; and drafting and/or revision of the manuscript. AG was a central MRI reader for safety in the bapineuzumab studies and contributed to interpretation of the data and drafting and/or revision of the manuscript. JBF was a central MRI reader for safety in the bapineuzumab studies and contributed to interpretation of the data and drafting and/or revision of the manuscript. DH contributed to the acquisition, analysis, and interpretation of data; drafting and/or revision of the manuscript for content; and study supervision and coordination. KL contributed to study supervision and coordination, analysis and interpretation of the data, and drafting and/or revision of the manuscript. DL carried out the primary statistical analysis for the 3000/3001 studies. He contributed to the study design, analysis and interpretation of the data, and drafting and/or revision of the manuscript. TM contributed to the design, analysis, and interpretation of data as well as drafting and/or revision of the manuscript. PL contributed to the study design; study supervision and coordination; acquisition, analysis, and interpretation of the data; and drafting and/or revision of the manuscript. IE contributed to the study design, analysis and interpretation of the data, and drafting and/or revision of the manuscript. KB contributed to study supervision and coordination and drafting and/or revision of the manuscript. GL contributed to acquisition, analysis, and interpretation of the data; study supervision and coordination; and drafting and/or revision of the manuscript. BTW contributed to study supervision and coordination, analysis and interpretation of the data, and drafting and/or revision of the manuscript. LH conducted protocol amendment and investigator's brochure updates and also contributed to data collection and review and to drafting and/or revision of the manuscript. LY contributed to the study design, analysis and interpretation of the data, and revision of the manuscript. HRB contributed to study concept and design; acquisition, analysis, and interpretation of data; drafting and/or revision of the manuscript; and study supervision and coordination. RSB contributed to the study design; acquisition, analysis, and interpretation of the data; and drafting and/or revision of the manuscript. All authors read and approved the final manuscript.

\section{Authors' information}

Additional author e-mail addresses: J. O. Rinne: juha.rinne@tyks.fi; M. Boada: mboada@fundacioace.com; S. Katayama: k-sada@msg.biglobe.ne.jp; P. Scheltens: p.scheltens@vumc.nl; B.Vellas: vellas.b@chu-toulouse.fr; M. Tuchman: mmtuchman@gmail.com; A. Gass: achim.gass@medma.uni-heidelberg.de; J.B. Fiebach: jochen.fiebach@charite.de; D. Hill: derek.hill@ixico.com; K. Lobello: kasia.lobello@pfizer.com; D. Li: david.li1@pfizer.com; T. McRae: thomas.mcrae@pfizer.com; P. Lucas: prisca.lucas@pfizer.com; I. Evans: iona.evans@pfizer.com; K. Booth: kevin.booth@pfizer.com; G. Luscan: gerald.luscan@pfizer.com; B.T.Wyman: bwyman2.0@gmail.com; L. Hua: yuelisa.hua@pfizer.com; L. Yang: lingfeng.yang@pfizer.com; H.R. Brashear: rbrashea@janimm.com; R. S. Black: ronald.black.md@gmail.com.

\section{Acknowledgements}

These studies were sponsored by Pfizer Inc. and Janssen Alzheimer Immunotherapy Research \& Development, LLC. The bapineuzumab 3000 and 3001 ClinicalTrials.gov identifiers are NCT00667810 and NCT00676143, respectively; the EudraCT identifiers are 2007-005994-79 and 2007-005995-14, respectively. Editorial and writing support was provided by Marsha L. Scott, Ph.D., at Phase Five Communications and was funded by Pfizer Inc. See Additional file 1 for a complete list of study investigators.

\section{Funding}

The study sponsors were Pfizer Inc. and Janssen Alzheimer Immunotherapy Research \& Development. 


\section{Author details}

${ }^{1}$ University Hospitals Leuven, Department of Neurosciences, Alzheimer Research Centre KU Leuven, Herestraat 49, 3000, Leuven, Belgium. ${ }^{2}$ Turku PET Centre and Division of Clinical Neurosciences, University of Turku and Turku University Hospital, Kiinamyllynkatu 4-8, 20520 Turku, Finland. ${ }^{3}$ Fundació ACE, Barcelona Alzheimer Treatment and Research Center, Gran via de Carles III, 85 Bis, 08028 Barcelona, Spain. ${ }^{4}$ Graduate School of Biomedical Sciences, Hiroshima University, 1-2-3 Kasumi, Minami-ku, Hiroshima 734-8551, Japan. ${ }^{5}$ Alzheimercentrum VUmc, Neurology, VU University Medical Center, PO Box 7057, 1007, MB, Amsterdam, Netherlands. ${ }^{6} \mathrm{CHU}$ Toulouse, Gérontopôle, 170 Avenue de Casselardit, TSA 40031, 31059 Toulouse, Cedex 9, France. ${ }^{7}$ Palm Beach Neurological Center, 3365 Burns Road, Suite 203, Palm Beach Gardens, FL 33410, USA. ${ }^{8}$ Department of Neurology, University Hospital Mannheim, University Medical Centre Mannheim, Theodor-Kutzer-Ufer 1-3, 68167 Mannheim, Germany. ${ }^{9}$ Center for Stroke Research Berlin (CSB), Charité Universitätsmedizin Berlin, Hindenburgdamm 30, 12200 Berlin, Germany. ${ }^{10}$ IXICO Ltd., The London Bioscience Innovation Centre, 4th Floor, Griffin Court, 15 Long Lane, London EC1A 9PN, UK. " 11 Pfizer Inc., 500 Arcola Road, Collegeville, PA 19426, USA. ${ }^{12}$ Pfizer Inc., 235 East 42nd Street, New York, NY 10017, USA. ${ }^{13}$ Pfizer Global Research and Development (PGRD), 23-25 avenue Du Docteur Lannelongue, Paris, Île-De-France 75014, France. ${ }^{14}$ Pfizer Ltd., Walton Oaks, Dorking Road, Tadworth, Surrey KT20 7NS, UK. ${ }^{15}$ Pfizer Inc., 7 Kings Highway, Groton, CT 06340, USA. ${ }^{16}$ Janssen Alzheimer Immunotherapy Research \& Development, LLC, 700 Gateway Boulevard, South San Francisco, CA 94080, USA.

Received: 3 February 2016 Accepted: 22 April 2016

Published online: 12 May 2016

\section{References}

1. Lemere CA. Immunotherapy for Alzheimer's disease: hoops and hurdles. Mol Neurodegener. 2013;8:36.

2. Tayeb HO, Murray ED, Price BH, Tarazi Fl. Bapineuzumab and solanezumab for Alzheimer's disease: is the 'amyloid cascade hypothesis' still alive? Expert Opin Biol Ther. 2013;13:1075-84.

3. Salloway S, Sperling R, Fox NC, Blennow K, Klunk W, Raskind M, et al. Two phase 3 trials of bapineuzumab in mild-to-moderate Alzheimer's disease. N Engl J Med. 2014;370:322-33.

4. Moreth J, Mavoungou C, Schindowski K. Passive anti-amyloid immunotherapy in Alzheimer's disease: what are the most promising targets? Immun Ageing. 2013;10:18.

5. Blennow K, Zetterberg H, Rinne JO, Salloway S, Wei J, Black R, et al. Effect of immunotherapy with bapineuzumab on cerebrospinal fluid biomarker levels in patients with mild to moderate Alzheimer disease. Arch Neurol. 2012;69: 1002-10.

6. Rinne JO, Brooks DJ, Rossor MN, Fox NC, Bullock R, Klunk WE, et al. ${ }^{11} \mathrm{C}$-PiB PET assessment of change in fibrillar amyloid- $\beta$ load in patients with Alzheimer's disease treated with bapineuzumab: a phase 2, double-blind, placebo-controlled, ascending-dose study. Lancet Neurol. 2010;9:363-72.

7. Salloway S, Sperling R, Gilman S, Fox NC, Blennow K, Raskind M, et al. A phase 2 multiple ascending dose trial of bapineuzumab in mild to moderate Alzheimer disease. Neurology. 2009;73:2061-70.

8. Blennow K, Hampel H, Zetterberg H. Biomarkers in amyloid- $\beta$ immunotherapy trials in Alzheimer's disease. Neuropsychopharmacology. 2014;39:189-201.

9. Doody RS, Thomas RG, Farlow M, Iwatsubo T, Vellas B, Joffe S, et al. Phase 3 trials of solanezumab for mild-to-moderate Alzheimer's disease. N Engl J Med. 2014;370:311-21.

10. Panza F, Solfrizzi V, Imbimbo BP, Giannini M, Santamato A, Seripa D, et al. Efficacy and safety studies of gantenerumab in patients with Alzheimer's disease. Expert Rev Neurother. 2014;14:973-86.

11. Liu E, Schmidt ME, Margolin R, Sperling R, Koeppe R, Mason NS, et al. Bapineuzumab 301 and 302 Clinical Trial Investigators. Amyloid- $\beta{ }^{11}$ C-PiB-PET imaging results from 2 randomized bapineuzumab phase $3 A D$ trials. Neurology. 2015;85:692-700.

12. Sperling RA, Jack CR Jr, Black SE, Frosch MP, Greenberg SM, Hyman BT, et al. Amyloid-related imaging abnormalities in amyloid-modifying therapeutic trials: recommendations from the Alzheimer's Association Research Roundtable Workgroup. Alzheimers Dement. 2011;7:367-85.

\section{Submit your next manuscript to BioMed Central and we will help you at every step:}

- We accept pre-submission inquiries

- Our selector tool helps you to find the most relevant journal

- We provide round the clock customer support

- Convenient online submission

- Thorough peer review

- Inclusion in PubMed and all major indexing services

- Maximum visibility for your research

Submit your manuscript at www.biomedcentral.com/submit 